\title{
DYNAMICAL MASSES AND MASS-TO-LIGHT RATIOS FOR GLOBULAR CLUSTERS
}

\author{
G. ILLINGWORTH
}

Kitt Peak National Observatory, Tucson, Arizona, U.S.A.*

\begin{abstract}
Dynamical masses have been determined for 10 globular clusters. Comparison of the dynamically determined $M / L$ values with those calculated from extrapolations of the observed upper main sequence/giant branch luminosity functions for $\mathrm{M} 3$ and $\mathrm{M} 5$ indicate that low mass stars $\left(\sim 0.2 M_{\odot}\right)$ comprise a large fraction of the total number.
\end{abstract}

The best means at present for obtaining data on the form of the luminosity function (and hence mass function) in globular clusters for the faint, unobservable low mass stars is from dynamical determinations of the cluster masses. The importance of the mass function is due to (i) the dependence of the dynamical evolution of the cluster upon it (see e.g. Prata, 1971; Spitzer and Hart, 1971) and (ii) the possibility of determining the initial mass function by the use of representative evolutionary models. A summary is given here of the results of an observational program to determine masses for 10 globular clusters. This is followed by a comparison of the dynamically determined $M / L$ values with those calculated from extrapolations to the observed luminosity functions of M3 and M5.

\section{Masses and $M / L$ Ratios}

Central velocity dispersions have been measured for 10 concentrated globular clusters from high dispersion spectra of the integrated light. These velocity dispersions are given in Table I. They were determined from the spectra by (i) comparing the

TABLE I

Observed data

\begin{tabular}{|c|c|c|c|c|c|}
\hline NGC & $\begin{array}{l}\left\langle V_{r}^{2}\right\rangle^{1 / 2} \\
\mathrm{~km} \mathrm{~s}^{-1}\end{array}$ & $\log \frac{r_{t}}{r_{c}}$ & $\begin{array}{l}r_{c} \\
\mathrm{pc}\end{array}$ & $\begin{array}{l}D \\
\mathrm{kpc}\end{array}$ & $M_{V}$ \\
\hline 104 & $10.5 \pm 0.4$ & 2.03 & 0.48 & $4.1 \pm 0.4$ & $-9.2 \pm 0.2$ \\
\hline 362 & $\begin{array}{ll}7.5 & 0.9\end{array}$ & 1.70 & 0.58 & $9.7 \quad 1.4$ & $\begin{array}{ll}-8.5 & 0.2\end{array}$ \\
\hline 1851 & 7.9 & 1.83 & 0.37 & 10.5 & -8.3 \\
\hline 2808 & $14.2 \quad 1.3$ & 1.75 & 0.73 & 10.0 & -9.7 \\
\hline 6093 & $12.5 \quad 2.5$ & 1.88 & 0.33 & $9.1 \quad 1.8$ & -8.1 \\
\hline 6266 & $13.7 \quad 1.1$ & 1.63 & 0.56 & $\begin{array}{ll}7.9 & 1.2\end{array}$ & $\begin{array}{ll}-9.3 & 0.3\end{array}$ \\
\hline 6388 & 18.9 & 1.75 & 0.50 & $11.6 \quad 2.3$ & $\begin{array}{ll}-9.6 & 0.4\end{array}$ \\
\hline 6441 & 17.6 & 1.70 & 0.41 & 9.3 & -8.9 \\
\hline 6715 & $14.2 \quad 1.0$ & 1.83 & 0.71 & 22.1 & -9.6 \\
\hline 6864 & 10.3 & 1.82 & 0.53 & $20.0 \quad 2.8$ & $\begin{array}{ll}-8.5 & 0.3\end{array}$ \\
\hline
\end{tabular}

* Operated by the Association of Universities for Research in Astronomy, Inc.. under contract with the National Science Foundation. 
globular cluster spectra with artificially broadened spectra of suitable comparison stars and (ii) comparison in the Fourier domain of the power spectra of the globular cluster spectra and comparison star spectra. Determination of the velocity dispersion with high accuracy was possible using the latter method. The parameters $r_{c}$ (the core radius) and $r_{t} / r_{c}$ (the ratio of the tidal and core radii) required for use of the selfconsistent models of King (1966) were obtained from photoelectric surface photometry and star counts. $r_{c}$ and $\log \left(r_{t} / r_{c}\right)$ are tabulated in Table I. In addition the distances and integrated magnitudes used are also given in Table $I$. The masses and $M / L$ ratios calculated from the models using the data in Table I are given in Table II. More

TABLE II

\begin{tabular}{rllll}
\multicolumn{3}{c}{ Masses and mass-to-light ratios } \\
\hline NGC & \multicolumn{2}{l}{ Mass } & $\left(M / L_{v}\right)_{\odot}$ \\
& $\left(M_{\odot} \times 10^{-6}\right)$ & & \\
\hline 104 & $0.54 \pm 0.07$ & & \multicolumn{2}{c}{$1.4 \pm 0.2$} \\
362 & 0.19 & 0.06 & 0.9 & 0.3 \\
1851 & 0.16 & 0.05 & 0.9 & 0.3 \\
2808 & 0.92 & 0.24 & 1.4 & 0.4 \\
6093 & 0.39 & 0.18 & 2.8 & 1.3 \\
6266 & 0.55 & 0.14 & 1.3 & 0.4 \\
6388 & 1.12 & 0.26 & 2.0 & 0.5 \\
6441 & 0.74 & 0.18 & 2.6 & 0.6 \\
6715 & 1.03 & 0.23 & 1.8 & 0.4 \\
6864 & 0.40 & 0.13 & 1.9 & 0.6
\end{tabular}

extensive data, details of the methods used and of the sources of error are given in Illingworth and Freeman (1974), Illingworth (1975), and Illingworth and Illingworth (1975).

\section{Luminosity Functions}

The mean $M / L$ value for the clusters in Table II is

$$
\left\langle M / L_{v}\right\rangle_{\odot}=1.7 \text {. }
$$

This is a convenient dynamical value for comparison with $M / L$ values derived from observed luminosity functions extrapolated to the unobservable faint low mass stars. The use of this mean $M / L$ is not to imply that all these clusters are expected to have the same $M / L$. Differences in the dynamical evolution between clusters will quite likely result in different present $M / L$ values even if the initial mass function was the same for all clusters.

The observed luminosity functions of M3 (Sandage, 1957) and M5 (Simoda and Tanikawa, 1972) have been chosen as representative of globular cluster upper main sequence/giant branch luminosity functions. The differences between these two functions probably stem from the methods used to extrapolate the counted numbers of stars in the outer regions to total numbers for the whole cluster. The need to use a dynamical model for this extrapolation has been pointed out by King and Wilson 
(1972) since stars of different mass are distributed differently in the cluster. As luminosity functions properly corrected for this effect are not yet available those for M3 and M5 will be used as they represent the present extremes in the ratio of main sequence/giant branch star numbers (see Figure 2 of Simoda and Tanikawa).

The first extrapolation used was to fit the solar neighborhood luminosity function recently described by Wielen (1974). This is shown in Figures 1a and 1b. The apparent distance moduli used were:

$$
\begin{array}{lll}
\text { M3: } & (m-M)_{\mathrm{app}, V}=14.83 & \text { Sandage (1970) } \\
\text { M5: } & (m-M)_{\mathrm{app}, V}=14.39 & \text { Arp (1962). }
\end{array}
$$

The solar neighborhood curve has been normalized to the luminosity functions at $M_{V} \sim+6$. The Wielen function is similar to the combined van Rhijn (1936), Luyten (1939), and Kuiper (1942) luminosity function used by Sandage (1957) as an extension to the observed M3 luminosity function. The large, disk red dwarf population discussed by Weistrop (1972) is not included in the Wielen function. For M5 the Wielen function was fitted to the data compensated for the effects of equipartition, as is shown by a dotted line. The uncompensated curve is shown by a broken line. The peaking of the compensated curve at $M_{V} \sim+6$ indicates that the compensation was probably too extreme but still an improvement over the uncompensated function.

To determine the probable number of white dwarf members the Sandage (1957) modified Salpeter (1955) initial luminosity function has been fitted above the turnoff point. All stars having a mass greater than the current turnoff/red giant mass were

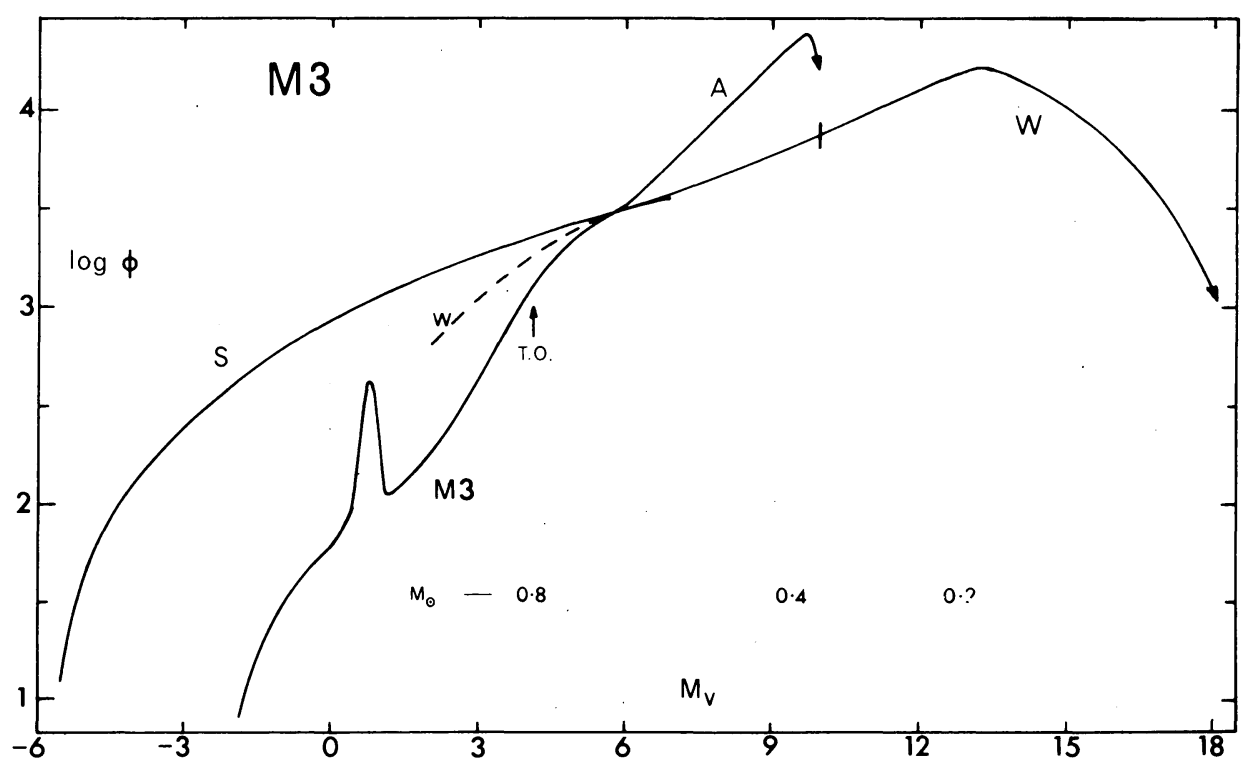

Fig. 1a. The observed luminosity functions of M3 and M5 fitted with (W) the Wielen luminosity function for the solar neighborhood, (S) the Sandage modified Salpeter initial luminosity function, (A) an arbitrarily increased function with mass cutoff at $M \sim 0.4 M_{\odot}$. 


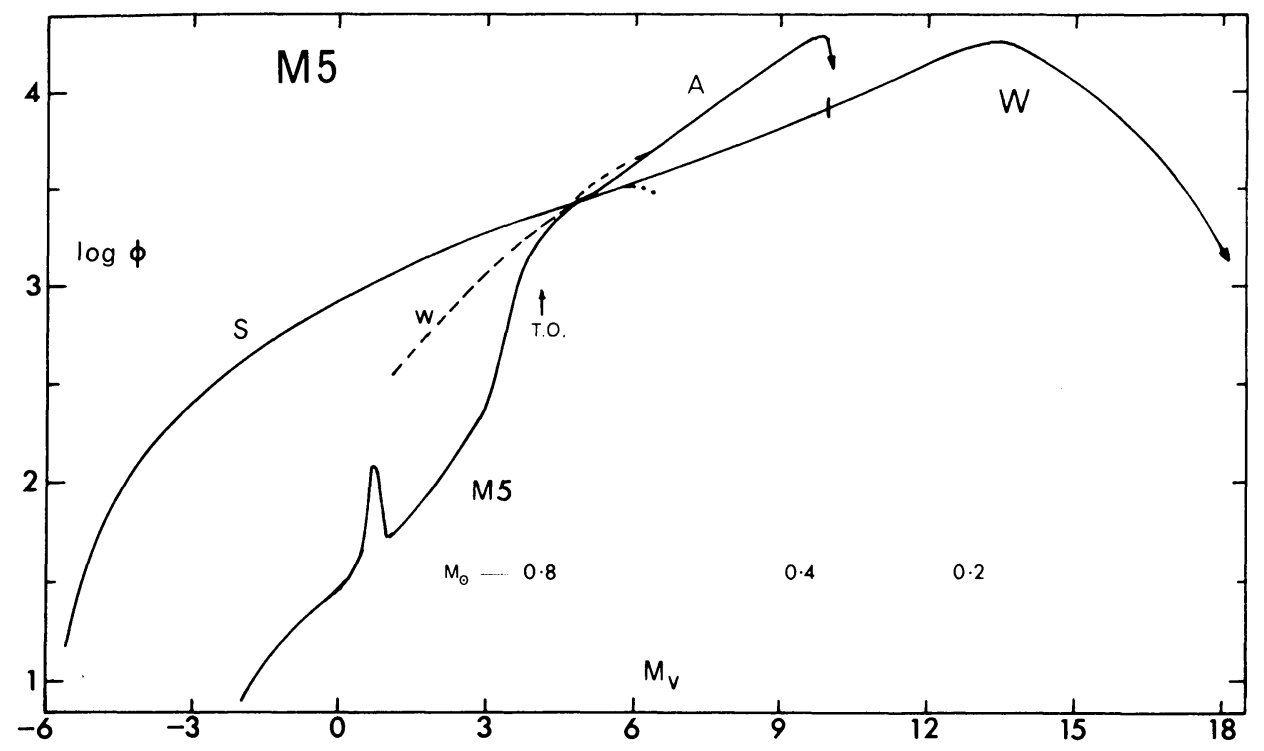

Fig. 1b. $\phi$ is the number of stars between $M_{v}+0.1$ and $M_{v}-0.1$. T.O. is the main sequence turnoff point. Further details are given in the text.

considered to have become white dwarfs. Prata (1971) found that the most likely interpretation of his results from an evolutionary study of M67 was that the initial mass function (for M67) was not a Salpeter function. The likely form was a power law with smaller slope (i.e. less low mass stars) at the low mass end but with a sharp increase in slope around 1.5-4 $M_{\odot}$. Thus the derived $M / L$ values below are given both with and without the white dwarfs to show the effect of a sharply decreased white dwarf component.

The next step was to assign masses to the white dwarfs, the giant branch and the main sequence stars. The mass of the white dwarfs was taken from the recent results of Wickramasinghe and Strittmatter (1972): $\langle M\rangle \sim 0.6 M_{\odot}$, Shipman (1972): $\langle M\rangle \sim 0.5$ $M_{\odot}$ and Trimble and Greenstein (1972): $\sim 0.65 M_{\odot}<\langle M\rangle<\sim 0.87 M_{\odot}$. The adopted mass was $M_{\text {WD }}=0.6 M_{\odot}$.

The results from color magnitude diagrams down to the main sequence for M3, M13, M15 and M92 (Sandage, 1970) combined with the main sequence/giant branch star models of Iben and Rood (1970a) indicate that the red giant mass $M_{\mathrm{RG}} \sim 0.8 M_{\odot}$ (see also Iben, 1971). Böhm-Vitense and Szkody (1973) using the main sequence data for M15 and M92 from the same paper by Sandage find $M_{\mathrm{RG}} \sim 0.85 M_{\odot} . M_{\mathrm{RG}}=0.8$ $M_{\odot}$ was used here.

For the horizontal branch stars $M_{\mathrm{HB}}=0.6 M_{\odot}$ was adopted (Iben and Rood, 1970b; Iben, 1971).

For the upper main sequence, where the bolometric corrections are small, assigning masses was fairly easy. The brightening of the main sequence was considered. The lower main sequence masses are more uncertain. This is due to the lack of a well 
established mass-luminosity relation and the uncertainty in the bolometric corrections. For the bolometric corrections on the main sequence the data considered was from Harris (1963), Lamla (1965), Copeland et al. (1970), and Johnson (1966) with the spectral type $(B-V)$ relation from Blaauw (1963). A mean relation was adopted. Similarly a mean relation was adopted from the $M_{\mathrm{bol}}$-mass relations given by Harris et al. (1963), Eggen (1967), Copeland et al. (1970), and McCluskey and Kondo (1972). The mass of the stars at the peak in the luminosity distribution at $M_{V} \sim 13.5$ was $\sim 0.2 M_{\odot}$.

If some degree of equipartition of energy occurs in globular clusters then this, combined with the galactic tidal force and the effect of gravitational shocks, may well have led to the loss of most of the low mass stars in the cluster (see Ostriker et al., 1972). The effect of this on the $M / L$ values was estimated by arbitrarily cutting the Wielen function at $M \sim 0.4 M_{\odot}$. The $M / L$ values [Table III; (b)] determined from this were somewhat lower than the mean dynamical value.

A further example using the same cutoff mass but having a higher $M / L$ value is shown on Figures $1 \mathrm{a}$ and $1 \mathrm{~b}$. This arbitrarily increased function was the result of an attempt to retain the low mass cutoff with an $M / L$ nearer the mean dynamical value.

TABLE III

Mass-to-light ratios

\begin{tabular}{|c|c|c|c|c|}
\hline & \multicolumn{2}{|c|}{ No white dwarfs } & \multicolumn{2}{|c|}{ With white dwarfs } \\
\hline & $\left(M / L_{V}\right)_{\odot}$ & $\langle M\rangle$ & $\left(M / L_{V}\right)_{\odot}$ & $\langle M\rangle$ \\
\hline M3(a) & 1.0 & 0.29 & 1.2 & 0.32 \\
\hline (b) & 0.5 & 0.57 & 0.7 & 0.58 \\
\hline (c) & 0.9 & 0.52 & 1.2 & 0.53 \\
\hline M5(a) & 1.4 & 0.29 & 1.7 & 0.32 \\
\hline (b) & 0.7 & 0.57 & 1.0 & 0.58 \\
\hline (c) & 1.1 & 0.53 & 1.4 & 0.55 \\
\hline
\end{tabular}

The derived $\left(M / L_{V}\right)_{\odot}$ values are given in Table III for M3 and M5 with the extrapolations:

(a) Wielen luminosity function,

(b) Wielen luminosity function cutoff at $M \sim 0.4 M_{\odot}$,

(c) raised luminosity function with the same cutoff.

Results both with and without the white dwarfs are presented. The mean mass $\langle M\rangle$ (in $M_{\odot}$ ) is also given.

\section{Discussion}

Before the $M / L$ values derived from extrapolation of the observed luminosity functions are compared with the mean dynamical $M / L$ value some consideration needs to be given to uncertainties in the dynamical value. The major uncertainty arises from 
the use of a single mass model in the mass derivation. The likely result of using models (again with an isotropic velocity distribution function like that of the single mass models) covering a range of stellar masses will be to increase the mass somewhat but probably by a factor less than 2 . Given this, the mean $\left\langle M / L_{V}\right\rangle_{\odot}=1.7$ is then underestimated. Thus it is likely that case (b) in Table III (Wielen luminosity function cutoff at $0.4 M_{\odot}$ ) is incorrect. Case (c), the raised luminosity function cutoff at $0.4 M_{\odot}$ appears unlikely as well since the disagreement with the observed function around $M_{V}=+6$ for both M3 and M5 is large and will get worse for higher $M / L$ values although the luminosity functions are rather uncertain at this point due to the effects of mass differentiation in the cluster. In the case of M3 inclusion of these effects will be such as to worsen the disagreement (note the compensated and uncompensated curves for M5 in Figure 1b).

Thus from the luminosity functions considered here the most likely extrapolation to the observed luminosity functions appears to be one similar to the solar neighborhood dwarf luminosity function. This means that stars of low mass (i.e. around $0.2 M_{\odot}$ ) still comprise a large fraction of the total number. A low mass cutoff from preferential depletion of low mass stars due to dynamical evolution is still possible but it will be closer to $0.2 M_{\odot}$ (or less) than $0.4 M_{\odot}$. Unless the number of white dwarfs is considerably larger than expected from the Salpeter initial mass function, they will not have an appreciable effect on these conclusions.

Definitive resolution, however, of the form of the mass function for low mass stars and of the number and mass of the white dwarfs will require a very detailed observational study (plus use of models covering a range of masses) of one or two clusters.

\section{Acknowledgements}

The observations reported here were carried out at Mt. Stromlo Observatory. I would like to thank the staff, in particular Ken Freeman, for their assistance. I am grateful for the use of the facilities at Mt. Stromlo and Siding Spring and for the assistance of an Australian National University Scholarship.

\section{References}

Arp, H. C.: 1962, Astrophys. J. 135, 311.

Blaauw, A.: 1963, Stars and Stellar Systems 3, 383.

Böhm-Vitense, E. and Szkody, P.: 1973, Astrophys. J. 184, 211.

Copeland, H., Jensen, J. O., and Jørgensen, H. E. : 1970, Astron. Astrophys. 5, 12.

Eggen, O. J.: 1967, Ann. Rev. Astron. Astrophys. 5, 105.

Harris, D. L., III. : 1963, Stars and Stellar Systems 3, 263.

Harris, D. L., III., Strand, K. Aa., and Worley, C. E.: 1963, Stars and Stellar Systems 3, 273.

Iben, I., Jr.: 1971, Publ. Astron. Soc. Pacific 83, 697.

Iben, I., Jr. and Rood, R. T.: 1970a, Astrophys. J. 159, 605.

Iben, I., Jr. and Rood, R. T. : 1970b, Astrophys. J. 161, 587.

Illingworth, G.: 1975, 'The Masses of Globular Clusters. II : Velocity Dispersions and Mass-to-Light Ratios', Astrophys. $J$. (in press).

Illingworth, G. and Freeman, K. C. : 1974, Astrophys. J. Letters 188, L83. 
Illingworth, G. and Illingworth, W.: 1975, 'The Masses of Globular Clusters. I: Surface Brightness Distributions and Star Counts', Astrophys. J. Suppl. (in press).

Johnson, H. L.: 1966, Ann. Rev. Astron. Astrophys. 4, 193.

King, I. R. : 1966, Astron. J. 71, 64.

King, I. R. and Wilson, C. P.: 1972, in A. G. Davis Philip (ed.), The Evolution of Population II Stars (Dudley Observatory Report, No. 4), p. 29.

Kuiper, G. P.: 1942, Astrophys. J. 95, 201.

Lamla, E. : 1965, in H. H. Voigt (ed.), Landolt-Bornstein, Springer-Verlag, Berlin, 1, p. 369.

Luyten, W.: 1939, Publ. Minnesota Obs. 2, No. 7, 123.

McCluskey, G. E., Jr. and Kondo, Y.: 1972, Astrophys. Space Sci. 17, 134.

Ostriker, J. P., Spitzer, L., Jr., and Chevalier, R. A.: 1972, Astrophys. J. Letters 176, L51.

Prata, S. W.: 1971, Astron. J. 76, 1017.

Rhijn, P. J., van: 1936, Groningen Publ. No. 47.

Salpeter, E. E.: 1955, Astrophys. J. 121, 161.

Sandage, A.: 1957, Astrophys. J. 125, 422.

Sandage, A.: 1970, Astrophys. J. 162, 841.

Shipman, H. L.: 1972, Astrophys. J. 177, 723.

Simoda, M. and Tanikawa, K.: 1972, Publ. Astron. Soc. Japan 24, 1.

Spitzer, L., Jr. and Hart, M. H.: 1971, Astrophys. J. 166, 483.

Trimble, V. and Greenstein, J. L. : 1972, Astrophys. J. 177, 441.

Weistrop, D. : 1972, Astron. J. 77, 849.

Wielen, R.: 1974, in G. Contopoulos (ed.), Highlights of Astronomy, Vol. 3, D. Reidel Publ. Co., Dordrecht-Holland, p. 395 .

Wickramasinghe, D. T. and Strittmatter, P..A. : 1972, Monthly Notices Roy. Astron. Soc. 160, 421.

\section{DISCUSSION}

King: This is a beautiful example of a new observational technique. As for the conclusions, I should like to emphasize that the main weight of the results goes into the number of low-mass stars which are too faint to observe in any other way. 